\title{
On Weak concircular Symmetries of Lorentzian Concircular Structure Manifolds
}

\author{
DHRUWA NARAIN \\ D.D.U.Gorakhpur University, \\ Department of Mathematics \& Statistics, \\ Gorakhpur, India. \\ profdndubey@yahoo.co.in
}

\author{
SUNIL YADAV \\ Alwar Institute of Engineering \& Technology, \\ Department of Mathematics, \\ Matsya Industrial Area, Alwar-301030, \\ Rajasthan, India. \\ prof_sky16@yahoo.com
}

\begin{abstract}
The object of the present paper is to study weakly concircular symmetric, weakly concircular Ricci symmetric and special weakly concircular Ricci symmetric Lorentzian concircular structure manifolds.
\end{abstract}

\section{RESUMEN}

El objetivo del presente artículo es estudiar las variedades de estructura simétricas concirculares débiles, las simétricas Ricci concirculares débiles y concirculares Lorentzianas simétricas Ricci concirculares débiles especiales.

Keywords and Phrases: Weakly concircular symmetric manifold, weakly concircular Ricci symmetric manifold, concircular Ricci tensor, special weakly concircular Ricci symmetric and Lorentzian concircular structure manifold.

2010 AMS Mathematics Subject Classification: 53C10, 53C15, 53C25. 


\section{Introduction}

The notion of weakly symmetric manifolds was introduced by Tamassy and Binh [8].A non-flat Riemannian manifold $\left(M^{n}, g\right)(n>2)$ is called weakly symmetric manifold if its curvature tensor $\mathrm{R}$ of type $(0,4)$ satisfies the condition

$$
\begin{aligned}
& \left(\nabla_{X} R\right)(Y, Z, U, V)=A(X) R(Y, Z, U, V)+B(Y) R(X, Z, U, V) \\
& +H(Z) R(Y, X, U, V)+D(U) R(Y, Z, X, V)+E(V) R(Y, Z, U, X)
\end{aligned}
$$

for all vector fields $X, Y, Z, U, V \in \chi\left(M^{n}\right), \chi(M)$ being the Lie-algebra of the smooth vector fields of $M$, where $A, B, H, D$ and $E$ are 1 -forms (not simultaneously zero) and $\nabla$ denote the operator of the covariant differentiations with respect to Riemannian metric $\mathrm{g}$. The 1 -forms are called the associated 1 -forms of the manifold and $n$-dimensional manifold of this kind is denoted by $(\text { WS })_{n}$. In 1999, De and Bandyopadhyay [2] studied a $(\mathrm{WS})_{\mathrm{n}}$ and prove that in such a manifold the associated 1 -forms $\mathrm{B}=\mathrm{H}$ and $\mathrm{D}=\mathrm{E}$. Hence from (1.1) reduces to the following:

$$
\begin{aligned}
& \left(\nabla_{X} R\right)(Y, Z, U, V)=A(X) R(Y, Z, U, V)+B(Y) R(X, Z, U, V) \\
& +B(Z) R(Y, X, U, V)+D(U) R(Y, Z, X, V)+D(V) R(Y, Z, U, X) .
\end{aligned}
$$

A transformation of $n$-dimensional Riemannian manifold $M$, which transform every geodesic circle of $M$ into a geodesic circle, is called a concircular transformation [11].The intersecting invariant of a concircular transformation is the concircular curvature tensor $\tilde{C}$ which is defined by [11].

$$
\tilde{C}(Y, Z, U, V)=R(Y, Z, U, V)-\frac{k}{n(n-1)}[g(Z, U) g(Y, V)-g(Y, U) g(Z, V)]
$$

where $k$ is the scalar curvature of the manifold.

Recently Shaikh and Hui [5] introduced the notion of weakly concircular symmetric manifolds. A Riemannian manifold is called weakly concircular symmetric manifold if its concircular curvature tensor $\tilde{\mathrm{C}}$ of type $(0,4)$ is not identically zero and satisfies the condition

$$
\begin{aligned}
& \left(\nabla_{X} \tilde{C}\right)(Y, Z, U, V)=A(X) \tilde{C}(Y, Z, U, V)+B(Y) \tilde{C}(X, Z, U, V) \\
& +H(Z) \tilde{C}(Y, X, U, V)+D(U) \tilde{C}(Y, Z, X, V)+E(V) \tilde{C}(Y, Z, U, X)
\end{aligned},
$$

for all vector fields $X, Y, Z, U, V \in \chi\left(M^{n}\right)$ where $A, B, H, D$ and $E$ are 1 -form (not simultane-

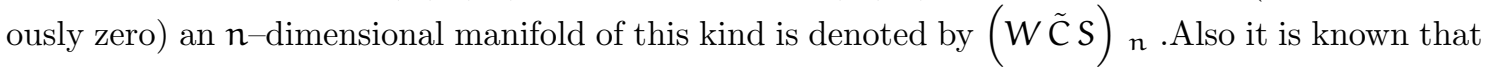
$[5]$, in a $(W \tilde{C} S)_{n}$ the associated 1 -forms $B=H$ and $D=E$, and hence the defining the condition (1.4) of a $(W, \tilde{C} S)_{n}$ reduces to the following form:

$$
\begin{aligned}
& \left(\nabla_{X} \tilde{C}\right)(Y, Z, U, V)=A(X) \tilde{C}(Y, Z, U, V)+B(Y) \tilde{C}(X, Z, U, V) \\
& +B(Z) \tilde{C}(Y, X, U, V)+D(U) \tilde{C}(Y, Z, X, V)+D(V) \tilde{C}(Y, Z, U, X)
\end{aligned},
$$


where $A, B$ and $D$ are 1 -forms (not simultaneously zero).

Again Tamassy and Binh [9] introduced the notion of weakly Ricci symmetric manifolds. A Riemannian manifold $\left(M^{n}, g\right),(n>2)$ is called weakly Ricci symmetric manifold if its Ricci tensor $S$ of type $(0,2)$ is not identically zero and satisfies the condition:

$$
\left(\nabla_{X} S\right)(Y, Z)=A(X) S(Y, Z)+B(Y) S(X, Z)+D(Z) S(Y, X)
$$

where $A, B$ and $D$ are three non-zero 1 -forms called the associate 1-forms of the manifold, and $\nabla$ is the operator of covariant differentiation with respect to metric $g$. Such $n$-dimensional manifold is denoted by (WRS) $n$. If $A=B=D$ then is called pseudo Ricci symmetric.

Let $\left\{e_{i}: i=1,2, \ldots . . . n\right\}$ be an orthonormal basis of the tangent space at each point of the manifold and let

$$
\tilde{S}(Y, V)=\sum_{i=1}^{n} \tilde{C}\left(Y, e_{i}, e_{i}, Y\right)
$$

Then from (1.3), we have

$$
\tilde{S}(Y, V)=S(Y, V)-\frac{k}{n} g(Y, V),
$$

The tensor $\tilde{S}$ is called the concircular Ricci symmetric tensor which is symmetric tensor of type $(0,2)$. In [1] De and Ghose introduced the notion of weakly concircular Ricci symmetric manifolds. A Riemannian manifold $\left(M^{n}, g\right),(n>2)$ is called weakly concircular Ricci symmetric manifolds [1] if its concircular Ricci tensor $\tilde{S}$ of type $(0,2)$ is not identically zero satisfies the condition:

$$
\left(\nabla_{X} \tilde{S}\right)(Y, Z)=A(X) \tilde{S}(Y, Z)+B(Y) \tilde{S}(X, Z)+D(Z) \tilde{S}(Y, X)
$$

where $A, B$ and $D$ are three 1-form (not simultaneously zero).If $A=B=D$ then $M^{n}$ is called pseudo concircular Ricci symmetric. A Riemannian manifold is called special weakly Ricci symmetric manifold if

$$
\left(\nabla_{X} S\right)(Y, Z)=2 A(X) S(Y, Z)+A(Y) S(X, Z)+A(Z) S(Y, X)
$$

where $A$ is a $1-$ form and is defined by

$$
A(X)=g(X, \rho) .
$$

where $\rho$ is the associated vector field.

Motivated by above studied we define and syudy special weakly concircular Ricci symmetric manifold. An $\mathrm{n}$-dimensional Riemannian manifold is called special weakly concircular Ricci symmetric manifolds. If

$$
\left(\nabla_{X} \tilde{S}\right)(Y, Z)=2 A(X) \tilde{S}(Y, Z)+A(Y) \tilde{S}(X, Z)+A(Z) \tilde{S}(Y, X) .
$$

where $\mathrm{A}$ is a 1 -form and is defined by (1.10). 
An $(2 n+1)$-dimensional Lorentzian manifold $M$ is smooth connected Para contact Hausdorff manifold with Lorentzian metric $g$, that is, $M$ admits a smooth symmetric tensor field $g$ of type $(0,2)$ such that for each point $p \in M$, the tensor $g_{p}: T_{p} M \times T_{p} M \rightarrow R$ is a non degenerate inner product of signature $\left(-,+, \ldots . .+\right.$ ) where $T_{p} M$ denotes the tangent space of $M$ at $p$ and $R$ is the real number space. In a Lorentzian manifold $(M, g)$ a vector field $\rho$ defined by

$$
g(X, \rho)=A(X)
$$

for any vector field $X \in \chi(M)$ is said to be concircular vector field [5] if

$$
\left(\nabla_{X} A\right)(Y)=\alpha[g(X, Y)+\omega(X) A(Y)]
$$

where $\alpha$ is a non zero scalar function, $A$ is a 1 -form and $\omega$ is a closed 1 -form.

Let $M$ be a Lorentzian manifold admitting a unit time like concircular vector field $\xi$, called the characteristic vector field of the manifold. Then we have

$$
g(\xi, \xi)=-1
$$

Since $\xi$ is the unit concircular vector field, there exist a non zero 1-form $\eta$ such that

$$
g(X, \xi)=\eta(X)
$$

the equation (1.13) of the following form holds:

$$
\left(\nabla_{X} \eta\right)(Y)=\alpha[g(X, Y)+\eta(X) \eta(Y)] \quad(\alpha \neq 0)
$$

for all vector field $X, Y$, where $\nabla$ denotes the operator of covariant differentiation with respect to Lorentzian metric $g$ and $\alpha$ is a non zero scalar function satisfying

$$
\left(\nabla_{X} \alpha\right)=(X \alpha)=\rho \eta(X)
$$

where $\rho$ being a scalar function. If we put

$$
\phi X=\frac{1}{\alpha} \nabla_{X} \xi
$$

Then from (1.14) and (1.16), we have

$$
\phi^{2} X=X+\eta(X) \xi
$$

from which it follows that $\phi$ is a symmetric $(1,1)$-tensor. Thus the Lorentzian manifold $M$ together with unit time like concircular vector field $\xi$, it's associate 1 -form $\eta$ and $(1,1)$-tensor field $\phi$ is said to be Lorentzian concircular structure manifolds (briefly (LCS) $2 n+1$-manifold) [6]. In particular if $\alpha=1$, then the manifold becomes LP-Sasakian structure of Matsumoto [3]. 


\section{Lorentzian Concircular Structure manifolds}

A differentiable manifold $M$ of dimension $(2 n+1)$ is called (LCS) $2 n+1$-manifold if it admits a $(1,1)$ -tensor $\phi$, a contravarient vector field $\xi$, a covariant vector field $\eta$ and a Lorentzian metric $g$ which satisfy the following

$$
\begin{gathered}
\eta(\xi)=-1, \\
\phi^{2}=I+\eta \otimes \xi, \\
g(\phi X, \phi Y)=g(X, Y)+\eta(X) \eta(Y), \\
g(X, \xi)=\eta(X), \\
\phi \xi=0 \quad, \eta(\phi X)=0,
\end{gathered}
$$

for all $X, Y \in T M$. Also in a (LCS) $2 n+1$-manifold the following relations are satisfied [7].

$$
\begin{gathered}
\eta(R(X, Y) Z)=\left(\alpha^{2}-\rho\right)[g(Y, Z) \eta(X)-g(X, Z) \eta(Y)] \\
R(X, Y) \xi=\left(\alpha^{2}-\rho\right)[\eta(Y) X-\eta(X) Y] \\
R(\xi, X) Y=\left(\alpha^{2}-\rho\right)[g(X, Y) \xi-\eta(Y) X] \\
R(\xi, X) \xi=\left(\alpha^{2}-\rho\right)[\eta(X) \xi+X] \\
\left(\nabla_{X} \phi\right)(Y)=\alpha[g(X, Y) \xi+2 \eta(X) \eta(Y) \xi+\eta(Y) X] \\
S(X, \xi)=2 n\left(\alpha^{2}-\rho\right) \eta(X) \\
S(\phi X, \phi Y)=S(X, Y)+2 n\left(\alpha^{2}-\rho\right) \eta(X) \eta(Y),
\end{gathered}
$$

Definition 2.1 A Lorentzian concircular structure manifold is said to be $\eta$-Einstein if the Ricci operator Q satisfies

$$
\mathrm{Q}=\mathrm{aId}+\mathrm{b \eta} \otimes \xi
$$

where $\mathrm{a}$ and $\mathrm{b}$ are smooth functions on the manifolds, In particular if $\mathrm{b}=0$, then $\mathrm{M}$ is an Einstein manifold. 


\section{Main Results}

Definition 3.1 A Lorentzian concircular structure manifold $\left(\mathrm{M}^{2 \mathrm{n}+1}, \mathrm{~g}\right)(\mathrm{n}>1)$ is said to be weakly concircular symmetric if its concircular curvature tensor $\tilde{\mathrm{C}}$ of type $(0,4)$ satisfies $(1.5)$

Substituting $Y=V=e_{i}$ in (1.5) and taking summation over $i, 1 \leq i \leq 2 n+1$, we get

$$
\begin{aligned}
& \left(\nabla_{X} S\right)(Z, U)-\frac{d \kappa(X)}{n} g(Z, U)=A(X)\left[S(Z, U)-\frac{\kappa}{n} g(Z, U)\right]+B(Z)[S(X, U) \\
& -\frac{k}{n} g(X, U)+D(U)\left[S(X, Z)-\frac{\kappa}{n} g(X, Z)\right]+B(R(X, Z) U)+D(R(X, U) Z) \\
& -\frac{k}{n(n-1)}[\{B(X)+D(X) g(Z, U)-B(Z) g(X, U)-D(U) g(Z, X)]
\end{aligned}
$$

Again setting $X=Z=U=\xi$ in (3.1) and using (2.7)and (2.11), we have

$$
A(\xi)+B(\xi)+D(\xi)=\frac{d k(\xi)}{k-2 n^{2}\left(\alpha^{2}-\rho\right)}
$$

This leads to the following result.

Theorem 3.1. In a weakly concircular symmetric Lorentzian concircular structure manifold $\left(M^{2 n+1}, g\right)(n>$ 1) the relation (3.2) holds.

Corollary 3.1 In a weakly concircular symmetric Lorentzian concircular structure manifold $\left(M^{2 n+1}, g\right)(n>$ 1) the sum of 1-forms $\mathrm{A}, \mathrm{B}$ and $\mathrm{D}$ is zero everywhere if and only if the scalar curvature $\mathrm{K}$ of the manifold is constant.

Next, putting $X$ and $Z$ by $\xi$ in (3.1) and using (2.4), (2.7)and (2.11) we obtain

$$
\begin{aligned}
& \left(\nabla_{X} S\right)(\xi, U)-\frac{d \kappa(\xi)}{n} \eta(U)=[A(\xi)+B(\xi)]\left\{2 n\left(\alpha^{2}-\rho\right)-\frac{k}{n}\right\} \eta(U)+ \\
& {\left[\frac{k}{n}-2 n\left(\alpha^{2}-\rho\right)-\frac{k}{n(n-1)}+1\right] D(U)+D(\xi)\left[\left(\alpha^{2}-\rho\right)-\frac{k}{n(n-1)}\right] \eta(U) .}
\end{aligned}
$$

Also from (2.11), we have

$$
\left(\nabla_{\xi} \mathrm{S}\right)(\xi, \mathrm{U})=0
$$

In view of (3.2) and (3.4), equation (3.3) reduces to

$$
D(U)=\left[\frac{k+(n-1)\left\{k-2 n^{2}\left(\alpha^{2}-\rho\right)\right\}}{-k+(n-1)\left\{k-2 n^{2}\left(\alpha^{2}-\rho\right)\right\}}\right] \quad D(\xi) \eta(U) .
$$

Next setting $\mathrm{X}=\mathrm{U}=\xi$ in (3.1) and proceeding in the similar manner as above, we have

$$
B(Z)=\left[\frac{k-(n-1)\left\{k-2 n^{2}\left(\alpha^{2}-\rho\right)\right\}}{-k+(n-1)\left\{k-2 n^{2}\left(\alpha^{2}-\rho\right)\right\}}\right] B(\xi) \eta(Z),
$$


Again, substituting $Z=U=\xi(3.1)$, we obtain

$$
\begin{aligned}
& \left(\nabla_{X} S\right)(\xi, \xi)+\frac{d \kappa(X)}{n}=A(X)\left[\frac{k}{n}-2 n\left(\alpha^{2}-\rho\right)\right]+\left[\frac{k}{n(n-1)}-\left(\alpha^{2}-\rho\right)\right] \\
& \{B(X)+D(X)+(B(\xi)+D(\xi)) \eta(X)\}+\left[2 n\left(\alpha^{2}-\rho\right)-\frac{k}{n}\right]\{B(\xi)+D(\xi)\} \eta(X)
\end{aligned}
$$

On the other hand we have

$$
\left(\nabla_{\xi} S\right)(\xi, \xi)=\nabla_{X} S(\xi, \xi)-2 S\left(\nabla_{X} \xi, \xi\right),
$$

which yield by using (1.16) and (2.1) that.

$$
\left(\nabla_{\xi} S\right)(\xi, \xi)=-2 \mathfrak{n}\left(\alpha^{2}-\rho\right) \xi
$$

In view of (3.7) and (3.8), we get

$$
\begin{aligned}
& A(X)=\left[\frac{d k(X)-2 n^{2}\left(\alpha^{2}-\rho\right) \xi}{k-2 n^{2}\left(\alpha^{2}-\rho\right)}\right]-\left[\frac{k}{(n-1)\left\{\left(k-2 n^{2}\left(\alpha^{2}-\rho\right)\right\}\right.}\right] \\
& \{B(X)+D(X)\}-\{D(\xi)+B(\xi)\} \eta(X)-\left\{\frac{d k(\xi)}{k-2 n^{2}\left(\alpha^{2}-\rho\right)}-A(\xi)\right\} \eta(X)
\end{aligned}
$$

This leads to the following result.

Theorem 3.2. In a weakly concircular symmetric Lorentzian concircular structure manifold $\left(M^{2 n+1}, g\right)(n>1)$ the associated 1-forms D, B and A are given by (3.5) (3.6) and (3.9) respectively.

Definition 3.2 A Lorentzian concircular structure manifold $\left(M^{2 n+1}, \mathrm{~g}\right)(\mathrm{n}>1)$ is said to be weakly concircular Ricci symmetric if its concircular Ricci tensor $\mathbb{S}$ of type $(0,2)$ satisfies (1.8).

In view of (1.8) and (1.9) yield

$$
\begin{aligned}
& \left(\nabla_{X} S\right)(Y, Z)-\frac{d k(X)}{n} g(Y, Z)=A(X)\left[S(Y, Z)-\frac{k}{n} g(Y, Z)\right]+B(Y) \\
& {\left[S(X, Z)-\frac{k}{n} g(X, Z)\right]+D(Z)\left[S(X, Y)-\frac{k}{n} g(X, Y)\right]}
\end{aligned}
$$

Setting $X=Y=Z=\xi$ in above we get the relation (3.2). Hence we can state the following

Theorem 3.3. In a weakly concircular Ricci symmetric Lorentzian concircular structure manifold $\left(M^{2 n+1}, g\right)(n>1)$ the relations (3.2) holds

Corollary 3.2. In a weakly concircular Ricci symmetric Lorentzian concircular structure manifold $\left(M^{2 n+1}, g\right)(n>1)$ the sum of 1 -forms $A, B$ and $D$ is zero everywhere and only if the scalar curvature $k$ of the manifold is constant.

Now, taking $X$ and $Y$ by $\xi$ in (3.10), we have 


$$
\left(\nabla_{\xi} \mathrm{S}\right)(\xi, Z)-\frac{\mathrm{d} \kappa(X)}{\mathrm{n}} \eta(Z)=\{\mathrm{A}(\xi)+\mathrm{B}(\xi)\}+\mathrm{D}(\mathrm{Z})\left[\mathrm{S}(\xi, \xi)+\frac{\kappa}{\mathrm{n}}\right]
$$

In view of (3.2) and (3.4), equation (3.11) yields.

$$
D(Z)=\frac{-d k(\xi)}{k-2 n^{2}\left(\alpha^{2}-\rho\right)} \eta(Z)+\left[\frac{d k(\xi)}{k-2 n^{2}\left(\alpha^{2}-\rho\right)}-D(\xi)\right] \eta(Z),
$$

Again putting $X=Z=\xi$ in (3.11) and proceeding in a similar manner as above, we get

$$
\begin{gathered}
B(Y)=\frac{-d k(\xi)}{k-2 n^{2}\left(\alpha^{2}-\rho\right)} \eta(Y)+\left[\frac{d k(\xi)}{k-2 n^{2}\left(\alpha^{2}-\rho\right)}-B(\xi)\right] \eta(Y), \\
A(X)=\frac{-2 n^{2}\left(\alpha^{2}-\rho\right) \xi \eta(x)}{k-2 n^{2}\left(\alpha^{2}-\rho\right)}+\frac{d k(X)}{k-2 n^{2}\left(\alpha^{2}-\rho\right)}+\left[\frac{d k(\xi)}{k-2 n^{2}\left(\alpha^{2}-\rho\right)}-A(\xi)\right] \eta(X),
\end{gathered}
$$

This leads to the following result.

Theorem 3.4. In a weakly concircular Ricci symmetric Lorentzian concircular structure manifold $\left(M^{2 n+1}, g\right)(n>1)$ the associated 1-form D, B and A are given by (3.12) (3.13) and (3.14) respectively

Adding equations (3.12) (3. 13) and (3.14), using (3.3) we obtain

$$
A(X)+B(X)+D(X)=\frac{d k(X)-2 n^{2}\left(\alpha^{2}-\rho\right) \xi}{k-2 n^{2}\left(\alpha^{2}-\rho\right)}
$$

for any vector field $X$.

This leads to the following result.

Theorem 3.5. In a weakly concircular Ricci symmetric Lorentzian concircular structure manifold $\left(M^{2 n+1}, g\right)(n>1)$ the sum of the associated 1-form A, B and D is given by (3.15)

Corollary 3.3 There is no weakly concircular Ricci symmetric Lorentzian concircular structure manifold $\left(M^{2 n+1}, g\right)(n>1)$ unless the sum of the 1 -forms is everywhere zero if $d k(X)=2 n^{2}\left(\alpha^{2}-\right.$ $\rho) \xi$.

Also taking cyclic sum of (1.11), we get

$$
\begin{aligned}
\left(\nabla_{X} \tilde{S}\right)(Y, Z)+ & \left(\nabla_{Y} \tilde{S}\right)(Z, Y)+\left(\nabla_{Z} \tilde{S}\right)(X, Y)=4 A(X) \tilde{S}(Y, Z) \\
& +A(Y) \tilde{S}(X, Z)+A(Z) \tilde{S}(Y, X)
\end{aligned}
$$

Let $M^{2 n+1}$ admits a cyclic Ricci tensor. Then (3.16) reduces to 


$$
A(X) \tilde{S}(Y, Z)+A(Y) \tilde{S}(X, Z)+A(Z) \tilde{S}(Y, X)=0 .
$$

Taking $\mathbf{Z}=\xi$ in above and then using (1.7), (1.10) and (2.11), we obtain

$$
\left[2 n^{2}\left(\alpha^{2}-\rho\right)-\frac{\kappa}{n}\right]\{A(X) \eta(Y)+A(Y) \eta(X)\}+\eta(\rho) \mathbb{S}(X, Y)=0 .
$$

Again taking $\mathbf{Z}=\xi$ in (3.17), we get

$$
2 \eta(\rho) \eta(X)=A(X)
$$

Taking $X=\xi$ in (3.18)and using (1.7), we yields

$$
\eta(\rho)=0
$$

In view of (3.18) and (3.19), we get $A(X)=0, \forall X$.

This leads to the following result.

Theorem 3.6.If a special weakly concircular Ricci symmetric Lorentzian concircular structure manifold $\left(M^{2 n+1}, g\right)(n>1)$ admits Cyclic Ricci tensor then the 1-form A must vanishes.

Finally for Einstein manifold $\left(\nabla_{X} S\right)(Y, Z)=0$ and $S(Y, Z)=a g(Y, Z)$. Then (1.7) and (1.11), we get

$$
\begin{gathered}
-\frac{d \kappa(X)}{n} g(Y, Z)=2 A(X)\left[\left(a-\frac{k}{n}\right) g(Y, Z)\right]+A(Y)\left[\left(a-\frac{k}{n}\right) g(X, Z)\right] \\
+A(Z)\left[\left(a-\frac{\kappa}{n}\right) g(X, Y)\right]
\end{gathered}
$$

Plugging $\mathrm{Z}=\mathrm{X}=\mathrm{Y}=\xi$ in (3.20), we obtain that

$$
4 \eta(\rho)(a n-\kappa)=d \kappa(\xi)
$$

which implies that if $\kappa$ is constant then $\eta(\rho)=0$, that is $A(Y)=0, \forall Y$. Therefore we state the results

Theorem 3.7. A special weakly concircular Ricci symmetric Lorentzian concircular structure manifold $\left(M^{2 n+1}, g\right)(n>1)$ can not Einstein manifold if the scalar curvature of the manifold is constant.

Corollary 3.4.In a special weakly concircular Ricci symmetric Lorentzian concircular structure manifold $\left(M^{2 n+1}, g\right)(n>1)$ the 1-form $A$ is given by

$$
\left[A(\xi)=\frac{d k(\xi)-2 n^{2}\left(\alpha^{2}-\rho\right)}{2\left\{k-2 n^{2}\left(\alpha^{2}-\rho\right)+n\right\}} .\right.
$$


Received: January 2012. Accepted: October 2012.

\section{References}

[1] De,U.C. and Ghose,G.C.,On weakly concircular Ricci symmetric manifolds, South East Assian J. Math. and Math. Sci. 3(2)(2005), 9-15.

[2] De, U.C. and Bandyopadhya, S., On weakly symmetric Riemannian spaces, Publ.Math.Debrecen, 54/3-4,(1999), 377-381.

[3] Matsumoto, K, On Lorentzian paracontact manifolds,Bull of Yamagata Univ.Nat.Soci. 12 (1989)151-156.

[4] Narain, Dhruwa and Yadav, Sunil, On weakly symmetric and Weakly Ricci symmetric LPSasakian manifolds. African Journal of Mathematics \& compute sciences Research, 4(10) (2011), 308-312.

[5] Shaikh,A.A. and Hui,S.K.,On weakly concircular symmetric manifolds, Ann. Sti .Ale Univ.,Al. I .CUZA",Din Iasi,LV,f.1 (2009), 167-186.

[6] Shaikh, A.A., Lorentzian almost paracontact manifolds with structure of concircular type, Kyungpook Math.J.43 (2003), 305-314.

[7] Shaikh, A.A., Basu, T. and Eyasmin,S.,On the existence of $\phi$-recurrent (LCS $)_{n^{-}}$manifolds, Extracta Mathematicae, 231,(2008),305-314

[8] Tamasy,L. and Binh,T.Q.,On weakly symmetric and weakly projective symmetric Riemannian manifolds, Coll. Math. Soc., J.Bolyai, 56(1989), 663-670.

[9] Tamasy,L. and Binh,T.Q.,On weakly symmetries of Einstein and Sasakian manifolds, Tensor N.S., 53 (1993), 140-148.

[10] Yadav,S. and Suthar,D.L., On a quarter symmetric non-metric connections in a generalized co-symplectic manifolds, Global Journal of Science Frontier Research,10(9),(2011), 51-57.

[11] Yano, K., Concircular geometry I, concircularTransformathions, Proc. Imp. Acad. Tokyo, 16 (1940), 195-200.

[12] Yadav,Sunil, Dwivedi,P.K. and Suthar,Dayalal, On (L C S) $2 n+1$ - Manifolds Satisfying Certain Conditions on the Concircular Curvature Tensor, Thi Journal of Mathematics,(9)(2011),597603.

[13] Yadav,S., Suthar,D.L. and Srivastava,A.K, Some Results on $M\left(f_{1}, f_{2}, f_{3}\right)_{2 n+1}$ Manifolds. International Journal of Pure \& Applied Mathematics, 70(3) (2011), 415-423. 